\title{
Editorial
}

\section{Control, Stability, and Qualitative Theory of Dynamical Systems 2014}

\author{
Nazim I. Mahmudov, ${ }^{1}$ Mark A. McKibben, ${ }^{2}$ Sakthivel Rathinasamy, ${ }^{3}$ and Yong Ren ${ }^{4}$ \\ ${ }^{1}$ Department of Mathematics, Eastern Mediterranean University, Gazimagusa, North Cyprus, Mersin 10, Turkey \\ ${ }^{2}$ Department of Mathematics, West Chester University of Pennsylvania, 25 University Avenue, West Chester, PA 19383, USA \\ ${ }^{3}$ Department of Mathematics, Sungkyunkwan University, Suwon 440 746, Republic of Korea \\ ${ }^{4}$ Department of Mathematics, Anhui Normal University, Wuhu 241000, China
}

Correspondence should be addressed to Nazim I. Mahmudov; nazim.mahmudov@emu.edu.tr

Received 21 December 2014; Accepted 21 December 2014

Copyright (C) 2015 Nazim I. Mahmudov et al. This is an open access article distributed under the Creative Commons Attribution License, which permits unrestricted use, distribution, and reproduction in any medium, provided the original work is properly cited.

Dynamical systems play a crucial role in the mathematical modeling of phenomena across disciplines. Understanding issues concerning controllability, stability, and other qualitative aspects of such systems is important in enhancing our understanding of the mathematical models in which they arise. This issue brings together five manuscripts covering some important problems in control theory-deterministic and stochastic-that have applications to a wide variety of disciplines. Elements of the theory and techniques from dynamical systems, control and optimal control theory, stochastic analysis, and stochastic evolution equations are used throughout these papers. The results established in all articles in this issue have application in multiple disciplines, and they sometimes contain replicable numerical analysis components that could have broader applicability.

On the more abstract end of the spectrum, two papers explore theoretical issues arising in stochastic differential equations and control theory. Specifically, L. Xiaohua et al. noted that structural changes of large scale interconnected systems with expanding construction have negative impact on the stability of systems and provided an improved method for designing an organically structured controller and observer of the expanding construction. $\mathrm{H}$. Dai et al. established a weak limit theorem for a special case of operator fractional Brownian motions using martingale difference sequences. This result has application, for instance, in the context of multivariate time series and long-range dependencies, queueing system, and problems related to large deviations.

The notion of synchronization is very important in population dynamics, power systems, chemical process simulation, and automatic control. J. Feng et al. analyzed the outer synchronization between two complex networks with varying time delays. This work contributes to the growing literature in that direction.

Finally, on the more applied end of the spectrum, the remaining two papers were focused on the analysis of specific mathematical models. M. Chipofya et al. presented a solution to a problem concerning the stability and trajectory tracking of a quadrotor system, which is a helicopter with four equally spaced rotors. The dynamics are highly nonlinear, making the control problem very challenging to study. Simulations are provided to aid in visualizing the abstract results. $\mathrm{H}$. Li and $\mathrm{X}$. Zhao studied the asymptotic behavior of solutions for the coupled nonlinear Schrodinger lattice system, which can be viewed as a discretization model of a two-component system of time-dependent nonlinear Gross-Pitaevskii equations; the latter arise naturally in the mathematical modeling of the binary mixture of Bose-Einstein condensates with two different hyperfine states.

It is our hope that this compilation of papers will provide our readers and researchers with new ideas to continue 
similar lines of research in control, stability, and qualitative theory of dynamical systems.

Nazim I. Mahmudov

Mark A. McKibben

Sakthivel Rathinasamy

Yong Ren 


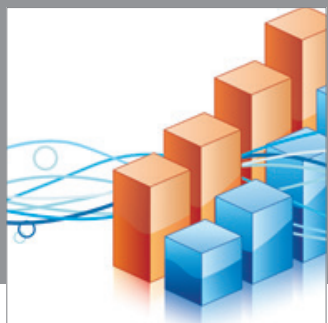

Advances in

Operations Research

mansans

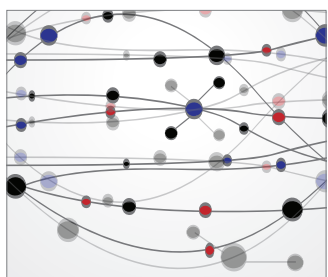

The Scientific World Journal
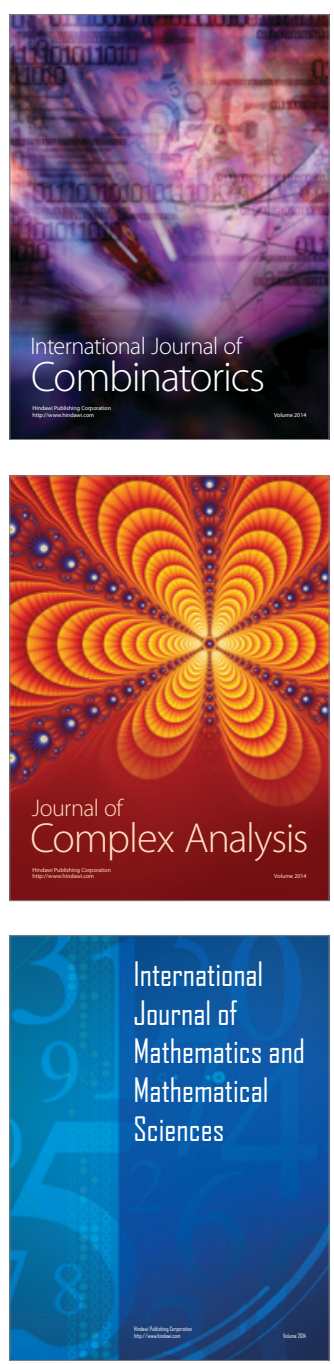
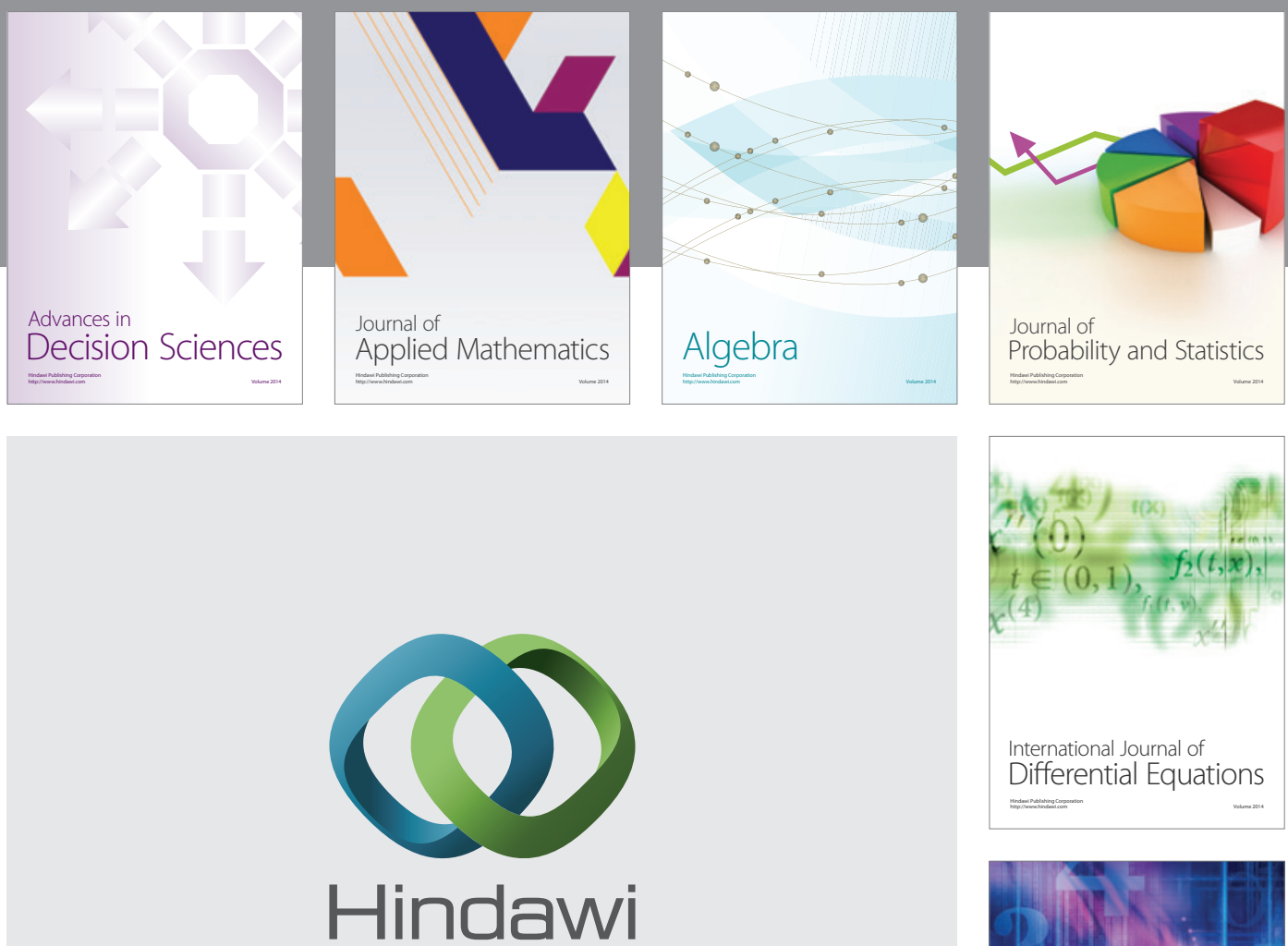

Submit your manuscripts at http://www.hindawi.com
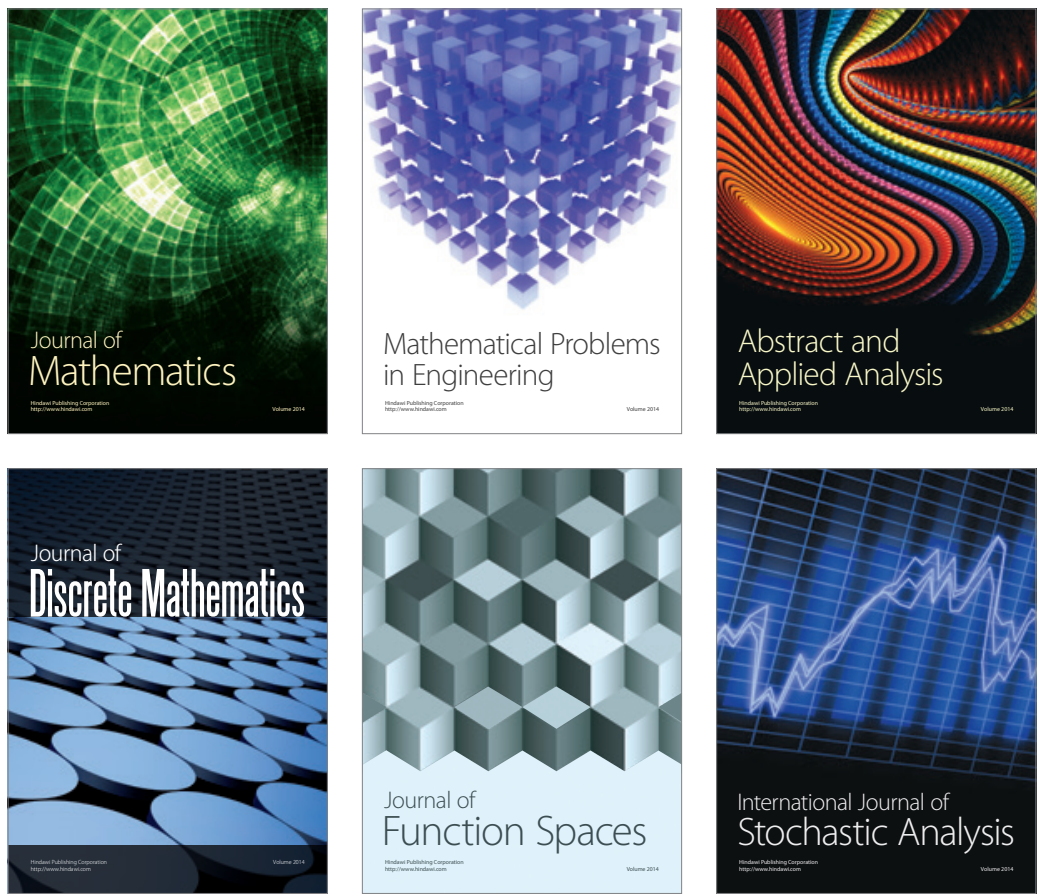

Journal of

Function Spaces

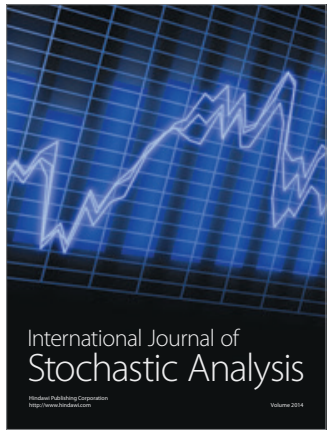

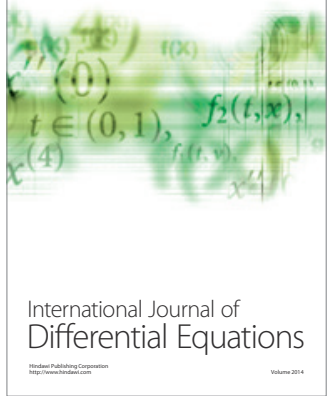
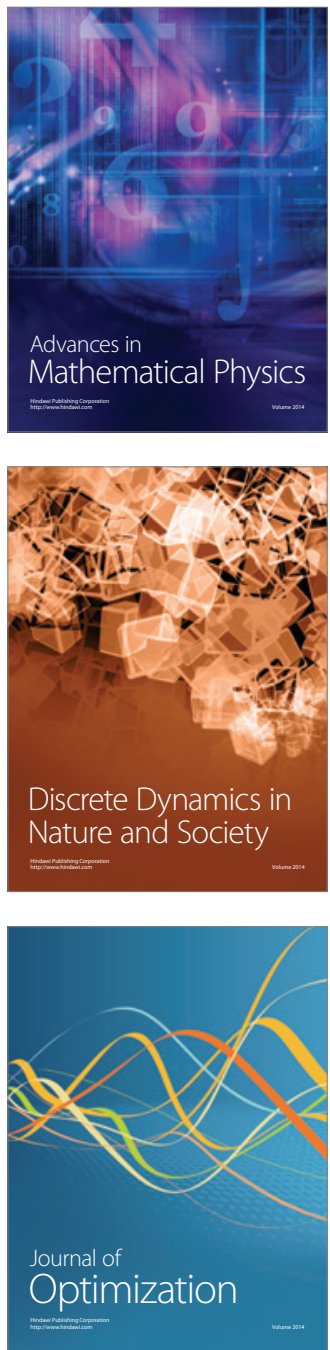